\title{
EXTREMALS AND TRANSVERSALITY OF THE GENERAL CALCULUS OF VARIATIONS PROBLEM OF THE FIRST ORDER IN SPACE*
}

BY

JESSE DOUGLAS $\dagger$

I. INTRODUCTION

The $\infty^{2}$ extremals of the calculus of variations problem

$$
\int F\left(x, y, y^{\prime}\right) d x=\text { minimum }
$$

may be identified with an arbitrarily given system of $\infty^{2}$ curves in the plane,

$$
y^{\prime \prime}=\phi\left(x, y, y^{\prime}\right),
$$

by solving for $F$ the partial differential equation

$$
F_{y}-F_{y^{\prime} x}-y^{\prime} F_{y^{\prime} y}-\phi F_{y^{\prime} y^{\prime}}=0 .
$$

We may say, in short, that apart from consisting of $\infty^{2}$ curves the system of extremals of the general problem (1) has no properties.

It is different in three dimensions. To identify the $\infty^{4}$ extremals of

$$
\int F\left(x, y, z, y^{\prime}, z^{\prime}\right) d x=\text { minimum }
$$

with a given system of $\infty^{4}$ curves defined by the differential equations

$$
\begin{aligned}
& y^{\prime \prime}=\phi\left(x, y, z, y^{\prime}, z^{\prime}\right), \\
& z^{\prime \prime}=\psi\left(x, y, z, y^{\prime}, z^{\prime}\right),
\end{aligned}
$$

we must find $F$ so as to satisfy simultaneously

$$
\begin{aligned}
F_{y}-F_{y^{\prime} z}-y^{\prime} F_{y^{\prime} y}-z^{\prime} F_{y^{\prime} z}-\phi F_{y^{\prime} y^{\prime}}-\psi F_{y^{\prime} z^{\prime}} & =0, \\
F_{z}-F_{z^{\prime} x}-y^{\prime} F_{z^{\prime} y}-z^{\prime} F_{z^{\prime} z}-\phi F_{y^{\prime} z^{\prime}}-\psi F_{z^{\prime} z^{\prime}} & =0 .
\end{aligned}
$$

This can not be done, in general. Thus a system of extremals of a problem of the form (4) must have properties which distinguish it in the totality of curve families definable by equations of the form (5). To express these

* Presented to the Society, February 26, 1927; received by the editors in August, 1926.

$\dagger$ National Research Fellow in Mathematics. 
properties analytically one would have to eliminate $F$ from the equations (6) and their implications, a procedure requiring an appalling amount of calculation.* Nor could the result be given any perspicuous geometric interpretation.

Besides its system of extremals, every problem of the form (4) defines a second geometric object-its transversality. This is a certain correspondence between lineal elements $\left(x, y, z, y^{\prime}, z^{\prime}\right)$ and surface elements $(x, y$, $z, p, q$ ) characterized by the following two properties: (A) a lineal element and its corresponding surface element have the same base point; (B) the $\infty^{2}$ extremals of (4) which are transverse to an arbitrary surface $\Sigma$ admit $\infty^{1}$ transverse surfaces, between any two of which arcs over which $\int F(x, y$, $\left.z, y^{\prime}, z^{\prime}\right) d x$ has the same value are intercepted on all these $\infty^{2}$ extremals.

Analytically, if the $p, q$ of a surface element are defined as the coefficients in the equation of its plane when written in the form

$$
\delta x+p \delta y+q \delta z=0,
$$

$\delta x, \delta y, \delta z$ denoting relative coördinates as to $x, y, z$, the surface element transverse to the lineal element $\left(x, y, z, y^{\prime}, z^{\prime}\right)$ is defined by

$$
p=\frac{F_{y^{\prime}}}{F-y^{\prime} F_{y^{\prime}}-z^{\prime} F_{z^{\prime}}}, \quad q=\frac{F_{z^{\prime}}}{F-y^{\prime} F_{y^{\prime}}-z^{\prime} F_{z^{\prime}}} .
$$

We shall use the term transversality to denote any correspondence between lineal and surface elements in which corresponding elements have the same base point. The general transversality is represented by two equations of the form

$$
p=p\left(x, y, z, y^{\prime}, z^{\prime}\right), \quad q=q\left(x, y, z, y^{\prime}, z^{\prime}\right),
$$

* Let $D_{r}^{5}$ denote the number of partial derivatives up to the $r$ th order inclusive of a function of five independent variables, the function itself being included as its derivative of zeroth order. The formula for $D_{r}^{5}$ is $(r+1)(r+2) \cdots(r+5) \div 5$ !. Then after forming all possible partial derivatives of the two equations (6) up to the $r$ th order inclusive, we have $2 D_{r}^{5}$ equations, which involve in a linear homogeneous way all the partial derivatives of $F$ to the order $r+2$ inclusive except the following: $F, F_{y^{\prime}}, F_{z^{\prime}}$, the derivatives such as $F_{x}, F_{x x}, \cdots$, involving $x$ alone up to the $(r+1)$ th order, and the derivatives of order $r+2$, involving only $x, y, z$. If $d_{r+2}^{3}(=(r+3)(r+4) \div 2)$ denote the number of partial derivatives of order $r+2$ of a function of three independent variables, the number of partial derivatives of $F$ to be eliminated from the $2 D_{r}^{5}$ equations is seen to be $D_{r+2}-d_{r+2}^{3}-(r+4)$. The number of equations remains less than the number of quantities to be eliminated until $r=11$, when these two numbers become respectively 8736,8448 . This indicate $s$ that we obtain by elimination 289 equations in the form of that number of determinants of order 8448 set equal to zero. These equations involve the derivatives of $\phi, \psi$ to the 11 th order inclusive, of which there are 4368 for each function. 
or inversely by

$$
y^{\prime}=y^{\prime}(x, y, z, p, q), \quad z^{\prime}=z^{\prime}(x, y, z, p, q),
$$

where $p, q$ and $y^{\prime}, z^{\prime}$ are arbitrary functions of their arguments.

Since (9) involves two arbitrary functions of five arguments, and (8) only one such function, it is obvious that a calculus of variations transversality must have special properties. These are expressed in analytic form by eliminating $F$ between the two equations (8). A geometric interpretation of the resulting partial differential equation in $p$ and $q$ has been given by E. Kasner, ${ }^{*}$ and is to the following effect.

In order that a transversality $T$ belong to a problem in the calculus of variations, it is necessary and sufficient that it be definable as follows: With each point $O$ of space associate, according to any (continuous, differentiable) law, a surface $\Sigma_{o}$. This may be done, and in the most general way, by laying off along each radius vector from $O$ a segment $O P$ whose length varies regularly from vector to vector, $\Sigma_{o}$ being the locus of points $P$. Then define the transverse surface element $\sigma$ to the lineal element $\lambda$ of $O P$ at $O$ to be the element in $O$ whose plane is parallel to the tangent plane of $\Sigma_{0}$ at $P$.

A transversality $T$ obtainable in the manner just described will be referred to in the statement of our main theorem as of special character.

We began by remarking that the characterization of the system of extremals of a variation problem (4) appears extremely difficult; and the transversality of such a problem has already been characterized. The object of the present paper is to characterize the composite of extremals and transversality.

In order to obtain a hint as to the nature of the characterization to be attempted, let us return to the property (B) of a variation transversality. The first part of (B), to the effect of the existence of $\infty^{1}$ transverse surfaces, is of purely geometric significance.

To each surface element $\sigma$ of $\Sigma$ let us construct the transverse lineal element $\lambda ; \lambda$ determines a definite extremal $C$, so that by varying $\sigma$ over $\Sigma$ we obtain the $\infty^{2}$ extremals transverse to $\Sigma$. Each of these has $\infty^{1}$ lineal elements, to each of which suppose constructed the transverse surface element. There arises in this way a field of $\infty^{3}$ surface elements (Pfaff equation). The significance of the first part of (B) is that this field of surface

* Transversality for double integrals in the calculus of variations and for contact transformations, an abstract of which appears in the Bulletin of the American Mathematical Society, vol. 21 (1914), pp. 71-72; see also p. 164. 
elements is integrable; its $\infty^{3}$ elements can be resolved into $\infty^{1}$ surfaces (exact Pfaff equation).

It is evident that the same considerations continue to apply when $\Sigma$ is any union-surface, curve, or joint.

The situation just described leads to the formulation of the following theorem, whose proof is the goal of the present paper.

THEOREM. If in connection with a family $\mathfrak{F}$ of $\infty^{4}$ curves in space definable analytically in the form (5) there exists a transversality $T$, necessarily of special character, such that the $\infty^{2}$ curves of $\mathfrak{F}$ which meet transversely an arbitrary base surface (union) $\Sigma$ admit $\infty^{1}$ transverse surfaces, then $\mathfrak{F}$ must be the system of extremals of a calculus of variations problem whose transversality is $T$.

This theorem is a generalization to the general problem (4) of a theorem of Kasner relative to problems of the important special form

$$
\int \nu(x, y, z) d s=\int \nu(x, y, z)\left(1+y^{\prime 2}+z^{\prime 2}\right)^{1 / 2} d x=\text { minimum } .
$$

It is readily shown that problems of the form (10) are characterized by the property that their transversality is orthogonality. Kasner proved* that if the $\infty^{2}$ curves of a given quadruply infinite family $\mathfrak{F}$ which meet an arbitrary surface $\Sigma$ orthogonally admit $\infty^{1}$ orthogonal surfaces (form a normal congruence), then $\mathfrak{F}$ must be the system of extremals of a problem of the form (10).

Our theorem admits of extension to $n$ dimensions without any difficulties besides those of calculation. In this form it includes a generalization by J. Lipka† of Kasner's theorem to any curved space of $n$ dimensions. For in a curved as well as in a flat space a problem whose transversality is orthogonality is necessarily of the form $\int \nu d s=$ minimum, where $\nu$ is a point function.

We may strengthen our theorem by requiring only that the property stated by it subsist for the following $5 \infty^{3}$ base manifolds instead of for an arbitrary $\Sigma$ : all points, all planes, any triple complex of curves. By the last we mean a system of $3 \infty^{3}$ curves such that an arbitrary pencil of space contains three distinct directions which belong to curves of the system.

* The theorem of Thomson and Tait and natural families of trajectories, these Transactions, vol. 11 (1910), pp. 121-140. See also Princeton Colloquium Lectures, 1909, published 1913, \$\$35-44. The results of the cited paper are extended in a paper by the present author, Normal congruences and quadruply infinite systems of curves in space, these Transactions, vol. 26 (1924), pp. 68-100.

$\dagger$ Some geometric investigations on the general problem of dynamics, Proceedings of the American Academy of Arts and Sciences, vol. 55 (1920), pp. 285-322. 
For instance, $3 \infty^{3}$ curves in horizontal planes do not form a triple complex in this sense, since if any pencil with non-horizontal plane be chosen, the directions of curves of the given $3 \infty^{3}$ which belong to this pencil coincide in its horizontal direction. Any three straight line complexes will serve.

\section{Duality Between the calculus of variations AND INFINITESIMAL CONTACT TRANSFORMATIONS}

We base our considerations on a certain duality between the calculus of variations and infinitesimal contact transformations (or one-parameter groups of contact transformations), which appears to have been first formulated by $\mathrm{E}$. Vessiot.* The present section is devoted to a presentation of this duality in a form suitable for application later in this paper-a form entirely different from that adopted by Vessiot.

Let us consider first the general infinitesimal surface element transformation $\epsilon$. It is defined by the equations

$$
\begin{gathered}
\delta x=\delta t X(x, y, z, p, q), \quad \delta y=\delta t Y(x, y, z, p, q), \delta t=\delta t Z(x, y, z, p, q), \\
\delta p=\delta t P(x, y, z, p, q), \quad \delta q=\delta t Q(x, y, z, p, q) .
\end{gathered}
$$

Under $\epsilon$ the base point of each element $\sigma,(x, y, z, p, q)$, receives an infinitesimal displacement in the direction $1: y^{\prime}: z^{\prime}$, where

$$
y^{\prime}=\frac{Y(x, y, z, p, q)}{X(x, y, z, p, q)}, \quad z^{\prime}=\frac{Z(x, y, z, p, q)}{X(x, y, z, p, q)} .
$$

We shall call the lineal element $\left(x, y, z, y^{\prime}, z^{\prime}\right)$ defined by (12) transverse $\dagger$ to $\sigma$. Thus every infinitesimal element transformation $\epsilon$ defines a certain transversality $T_{e}$.

The transformation $\epsilon$ also defines a system of $\infty^{4}$ curves $\mathfrak{F}_{\epsilon}$ called its path curves. Allow $\epsilon$ to operate repeatedly on an element $\sigma$. Then the locus of the base point of $\sigma$ is a curve termed a path curve $\ddagger$ of $\epsilon$. There are evidently $\infty^{4}$ such curves, since each of the $\infty^{5}$ elements $\sigma$ of space may be used to generate one, while each path curve absorbs $\infty^{1}$ elements $\sigma$. The differential equations of the path curves $\mathfrak{F}_{\epsilon}$ are

* Sur l'interprétation mécanique des transformations de contact infinitésimales, Bulletin de la Société Mathématique de France, vol. 34 (1906), pp. 230-269.

$\dagger$ This term has been proposed in connection with contact (not general) transformations of lineal elements by Kasner, The infinitesimal contact transformations of mechanics, Bulletin of the American Mathematical Society, vol. 16 (1910), pp. 408-412.

$\ddagger$ Vessiot uses the terms conjugué, trajectoire for transverse, path curve. 


$$
\begin{aligned}
& y^{\prime \prime}=\left(\frac{Y}{X}\right)_{x}+\frac{Y}{X}\left(\frac{Y}{X}\right)_{y}+\frac{Z}{X}\left(\frac{Y}{X}\right)_{z}+\frac{P}{X}\left(\frac{Y}{X}\right)_{p}+\frac{Q}{X}\left(\frac{Y}{X}\right)_{Q}, \\
& z^{\prime \prime}=\left(\frac{Z}{X}\right)_{x}+\frac{Y}{X}\left(\frac{Z}{X}\right)_{y}+\frac{Z}{X}\left(\frac{Z}{X}\right)_{z}+\frac{P}{X}\left(\frac{Z}{X}\right)_{p}+\frac{Q}{X}\left(\frac{Z}{X}\right)_{q},
\end{aligned}
$$

where in the second member we are to substitute for $p, q$ the functions of $x, y, z, y^{\prime}, z^{\prime}$ which result by solving (12) for $p, q$. Formula (13) is obtained by expanding

$$
y^{\prime \prime}=\frac{\delta y^{\prime}}{\delta x}=\frac{\delta\left(\frac{Y}{X}\right)}{\delta x}, \quad z^{\prime \prime}=\frac{\delta z^{\prime}}{\delta x}=\frac{\delta\left(\frac{Z}{X}\right)}{\delta x},
$$

with the use of (11).

It is evident from (12) and (13) that the transversality and path curves of $\epsilon$ depend only on the ratios $X: Y: Z: P: Q$.

Conversely, if a transversality $T$ and a quadruply infinite curve family $\mathfrak{F}$ are arbitrarily assigned, there exists an $\epsilon$ having $T$ for its transversality and $\mathfrak{F}$ for its path curves, this $\epsilon$ being determined up to multiplication of its defining functions $X, Y, Z, P, Q$ by a common factor $\rho(x, y, z, p, q)$, where $\rho$ is an arbitrary function. For $T$ determines $Y / X, Z / X$ by (12); further, since $\mathfrak{F}$ is given, say in the form (5), the left members of (13) are equal to known functions $\phi, \psi$ of $x, y, z, y^{\prime}, z^{\prime}$, therefore by the substitution (12) to known functions of $x, y, z, p, q$; thus $P / X, Q / X$ are uniquely determined, for the equations (13) are linear for $P / X, Q / X$, and their determinant, $(Y / X)_{p}(Z / X)_{q}-(Y / X)_{q}(Z / X)_{p}$ is not equal to zero, since, as we presume, the equations (12) which define $T$ are solvable for $p, q$.

As is well known, an element transformation is called a contact transformation when and only when it converts every union of elements $\sigma$ into a union of elements. The general infinitesimal contact transformation can be represented analytically in explicit form by means of its characteristic function $W(x, y, z, p, q)$ as follows:*

$$
\begin{array}{ll}
\delta x=\delta t\left(W-p W_{p}-q W_{q}\right), & \delta y=\delta t W_{p}, \quad \delta z=\delta t W_{q}, \\
\delta p=\delta t\left(p W_{x}-W_{y}\right), & \delta q=\delta t\left(q W_{x}-W_{z}\right) .
\end{array}
$$

We shall refer to (14) as the infinitesimal contact transformation $W$. Its transversality is defined by

- This representation, based on the form (7) $\delta x+p \delta y+q \delta z=0$ for the plane of an element $\sigma$, leads to more symmetrical formulas than the one given by Lie, Theorie der Transformationsgruppen, Leipzig, 1890, vol. 2, p. 252, and used by Vessiot, loc. cit., p. 230. Their representation is based on the form $p \delta x+q \delta y-\delta z=0$ for the plane of $\sigma$. 


$$
y^{\prime}=\frac{W_{p}}{W-p W_{p}-q W_{q}}, \quad z^{\prime}=\frac{W_{q}}{W-p W_{p}-q W_{q}} .
$$

Now, a problem of first order in the calculus of variations is defined by a function $F\left(x, y, z, y^{\prime}, z^{\prime}\right)$ of the lineal elements $\lambda$ of space; an infinitesimal contact transformation by a function $W(x, y, z, p, q)$ of the surface elements $\sigma$. To each $F$ we can make correspond a $W$ as follows. $F$ being given, each element $\lambda$ has a transverse element $\sigma$, defined by (11). Associate with $\sigma$ the value $W$ defined by the equation

$$
W F=1+y^{\prime} p+z^{\prime} q .
$$

In this way a function $W(x, y, z, p, q)$ is defined, which taken as characteristic function determines an infinitesimal contact transformation. We assert that the transversality and path curves of $W$ are identical respectively with the transversality and extremals of $F$.

Conversely, let the infinitesimal contact transformation $W$ be given. It causes to correspond to each element $\sigma$ a transverse element $\lambda$, defined by (15). Associate with $\lambda$ the value $F$ defined by (16). Then the variation problem defined by $F$ has the same transversality as $W$, and its extremals are identical with the path curves of $W$.

For the proof, we remark first that the equations (8) defining the transversality of $F$ may be combined into

$$
\frac{d F}{F}=\frac{p d y^{\prime}+q d z^{\prime}}{1+y^{\prime} p+z^{\prime} q}
$$

where $d F$ is formed on the supposition that the point $(x, y, z)$ is fixed. Similarly, the equations (15) defining the transversality of $W$ may be combined, on the same supposition, into

$$
\frac{d W}{W}=\frac{y^{\prime} d p+z^{\prime} d q}{1+y^{\prime} p+z^{\prime} q} .
$$

By logarithmic differentiation of $(16),(x, y, z)$ being regarded as fixed, we have

$$
\frac{d F}{F}+\frac{d W}{W}=\frac{p d y^{\prime}+q d z^{\prime}}{1+y^{\prime} p+z^{\prime} q}+\frac{y^{\prime} d p+z^{\prime} d q}{1+y^{\prime} p+z^{\prime} q} .
$$

It follows from (19) that (17) implies (18) and conversely. Thus if $\left(y^{\prime}, z^{\prime}\right)$ and $(p, q)$ correspond by the transversality of $F$, they correspond by the transversality of $W$, and conversely. 
The extremals of $F$ are defined by the differential equations

$$
\begin{aligned}
& F_{y}-\frac{d}{d x} F_{y^{\prime}}=0, \\
& F_{z}-\frac{d}{d x} F_{z^{\prime}}=0 .
\end{aligned}
$$

We have to prove that the same equations are satisfied by the path curves of $W$. From (8),

$$
\frac{F_{y^{\prime}}}{F}=\frac{p}{1+y^{\prime} p+z^{\prime} q}, \quad \frac{F_{z^{\prime}}}{F_{z^{\prime}}}=\frac{q}{1+y^{\prime} p+z^{\prime} q} ;
$$

therefore, by (16),

$$
F_{y^{\prime}}=\frac{p}{W}, \quad F_{z^{\prime}}=\frac{q}{W} .
$$

Thus, it has to be shown that along a path curve of $W$,

$$
\begin{aligned}
& F_{y}-\frac{\delta}{\delta x}\left(\frac{p}{W}\right)=0, \\
& F_{z}-\frac{\delta}{\delta x}\left(\frac{q}{W}\right)=0 .
\end{aligned}
$$

We have

$$
\begin{aligned}
\delta\left(\frac{p}{W}\right) & =\frac{W \delta p-p \delta W}{W^{2}} \\
& =\frac{1}{W^{2}}\left\{W \delta p-p\left(W_{z} \delta x+W_{\delta} \delta y+W_{\delta} \delta z+W_{p} \delta p+W_{\delta} \delta q\right)\right\} .
\end{aligned}
$$

By means of (14) the bracket reduces to $-W W_{y} \delta t$, so that

$$
\delta\left(\frac{p}{W}\right)=-\frac{W_{v}}{W} \delta t
$$

Again, by (14),

$$
\delta x=\delta t\left(W-p W_{q}-q W_{q}\right) .
$$

Making the substitutions (15) in (16), we find

$$
F=\frac{1}{W-p W_{q}-q W_{q}} ;
$$


hence

$$
\delta x=\frac{1}{F} \delta t .
$$

Substituting (25), (28) in the first equation (23), we obtain

$$
\frac{F_{y}}{F}+\frac{W_{y}}{W}=0 \text {. }
$$

Similarly the second equation (23) can be made to depend upon

$$
\frac{F}{F}+\frac{W}{W}=0
$$

The identity of the extremals of $F$ and the path curves of $W$ is established if we can prove (29), (30). To this end, let us form the logarithmic derivative of (16) partially with respect to $y$, from the point of view that the $p, q$ in $W$ and in the right member are functions of $x, y, z, y^{\prime}, z^{\prime}$ by means of the common transversality of $W$ and $F$. We find

$$
\text { (31) } \frac{F_{y}}{F}+\frac{W_{y}}{W}+\frac{W_{p}}{W} \frac{\partial p}{\partial y}+\frac{W_{q}}{W} \frac{\partial q}{\partial y}=\frac{y^{\prime}}{1+y^{\prime} p+z^{\prime} q} \frac{\partial p}{\partial y}+\frac{z^{\prime}}{1+y^{\prime} p+z^{\prime} q} \frac{\partial q}{\partial y} \text {. }
$$

By (15) the terms in $\partial p / \partial y, \partial q / \partial y$ cancel, giving

$$
\frac{F_{y}}{F}+\frac{W_{y}}{W}=0
$$

or (29). Similarly (30) can be proved. Thus the duality between $F$ and $W$ is established.

Suppose now that an infinitesimal element transformation $\epsilon$ is applied repeatedly to the $\infty^{2}$ elements of an arbitrary surface $\Sigma$. A field of $\infty^{3}$ surface elements is generated which may or may not be integrable. With the developments of the present section in mind we may reformulate our main theorem stated in the preceding section, as follows:

THEOREM. If the field of $\infty^{3}$ surface elements generated by applying a given infinitesimal element tranformation $\epsilon$ with infinite repetition to the elements of an arbitrary surface (union) $\Sigma$ is integrable, then $\epsilon$ must be proportional to an infinitesimal contact transformation $W$.

By the last phrase we mean that the defining functions $X, Y, Z, P, Q$ of $\epsilon$ are proportional to those of an infinitesimal contact transformation as they appear in (14).

The sequel is devoted to a proof of the theorem in the form just stated. 
III. CONDITIONS THAT AN INFINITESIMAL ELEMENT TRANSFORMATION BE PROPORTIONAL TO AN INFINITESIMAL CONTACT TRANSFORMATION

We prove in this section that, given $X, Y, Z, P, Q$, necessary and sufficient conditions that there exist a $\rho$ and a $W$ such that

$$
\begin{array}{ll}
X=\rho\left(W-p W_{p}-q W_{q}\right), & Y=\rho W_{p}, \quad Z=\rho W_{q}, \\
P=\rho\left(p W_{x}-W_{y}\right), & Q=\rho\left(q W_{x}-W_{z}\right),
\end{array}
$$

are the following; where

$$
M=X+p Y+q Z:^{*}
$$

(a)

$$
\begin{aligned}
& \left(\frac{Y}{M}\right)_{\mathrm{q}}=\left(\frac{Z}{M}\right)_{\mathrm{p}}, \\
& \left(\frac{P}{M}\right)_{\mathrm{q}}=p\left(\frac{Z}{M}\right)_{x}-\left(\frac{Z}{M}\right)_{y},
\end{aligned}
$$

(34) (c)

$$
\left(\frac{Q}{M}\right)_{p}=q\left(\frac{Y}{M}\right)_{x}-\left(\frac{Y}{M}\right)_{s}
$$

(d) $p\left(\frac{Y}{M}\right)_{x}-\left(\frac{Y}{M}\right)_{y}-\left(\frac{P}{M}\right)_{p}=q\left(\frac{Z}{M}\right)_{x}-\left(\frac{Z}{M}\right)_{x}-\left(\frac{Q}{M}\right)_{\mathrm{q}}$,

$$
q\left(\frac{P}{M}\right)_{x}-\left(\frac{P}{M}\right)_{z}=p\left(\frac{Q}{M}\right)_{x}-\left(\frac{Q}{M}\right)_{y} .
$$

To prove the conditions (34) necessary, or that (32) implies (34), we first combine (32) and (33) to obtain

$$
M=\rho W .
$$

Dividing (32) by (35), we have, with the notation

$$
\begin{aligned}
& V=\log W, \\
& \frac{X}{M}=1-p V_{p}-q V_{q}, \quad \frac{Y}{M}=V_{p}, \quad \frac{Z}{M}=V_{q}, \\
& \frac{P}{M}=p V_{x}-V_{y}, \quad \frac{Q}{M}=q V_{x}-V_{s} .
\end{aligned}
$$

The equations (34) were derived by elimination of $V$ from (37), and their necessity can be verified by direct substitution.

* We assume $M \neq 0$, that is, that the lineal element transverse to any given surface element does not lie in the plane of that element. 
To establish the sufficiency of the conditions (34), we begin by observing that (34) (a) implies the existence of a $\sigma(x, y, z, p, q)$ such that

$$
\frac{Y}{M}=\sigma_{p}, \quad \frac{Z}{M}=\sigma_{q},
$$

and (34) (e) the existence of a $\tau(x, y, z, p, q)$ such that

$$
\frac{P}{M}=p \tau_{x}-\tau_{y}, \quad \frac{Q}{M}=q \tau_{z}-\tau_{z},
$$

for (34) (e) can be written

$$
\left(q \frac{\partial}{\partial x}-\frac{\partial}{\partial z}\right) \frac{P}{M}=\left(p \frac{\partial}{\partial x}-\frac{\partial}{\partial y}\right) \frac{Q}{M},
$$

and the linear partial differential operators involved have coefficients constant as to the variables of differentiation.*

Substituting the values (38), (39) in (34) (b), (c), (d), we obtain

$$
\begin{array}{rlrl} 
& (41) & p \tau_{q x}-\tau_{q y} & =p \sigma_{q x}-\sigma_{q y}, \\
(42) & q \tau_{p x}-\tau_{p z} & =q \sigma_{p x}-\sigma_{p z}, \\
& (43) & \left(p \sigma_{p x}-\sigma_{p y}\right)-\left(p \tau_{p x}-\tau_{p y}\right) & =\left(q \sigma_{q x}-\sigma_{q z}\right)-\left(q \tau_{q x}-\tau_{q z}\right) .
\end{array}
$$

These can be written as equations in

$$
\omega=\tau-\sigma
$$

as follows:

(a)

$$
\left(p \frac{\partial}{\partial x}-\frac{\partial}{\partial y}\right) \omega_{q}=0,
$$

$$
\left(q \frac{\partial}{\partial x}-\frac{\partial}{\partial z}\right) \omega_{p}=0 \text {, }
$$

(c)

$$
\left(p \frac{\partial}{\partial x}-\frac{\partial}{\partial y}\right) \omega_{p}=\left(q \frac{\partial}{\partial x}-\frac{\partial}{\partial z}\right) \omega_{q},
$$

- The general solution of

$$
\sum b_{i} \frac{\partial}{\partial x_{i}} \phi=\sum a_{i} \frac{\partial}{\partial x_{i}} \psi,
$$

where the $a_{i}, b_{i}$ are constants, is

$$
\phi=\sum a_{i} \frac{\partial}{\partial x_{i}} \omega, \quad \psi=\sum b_{i} \frac{\partial}{\partial x_{i}} \omega,
$$

where $\omega$ is an arbitrary function of the variables $x$. 
of which we have to find the general solution. Equations (45) (a), (b) imply respectively

$$
\begin{aligned}
& \omega_{q}=\psi(\xi, z, p, q), \\
& \omega_{p}=\phi(\xi, y, p, q),
\end{aligned}
$$

where

$$
\xi=x+p y+q z .
$$

Substituting in (45) (c), we obtain

$$
p \phi_{\xi}-p \phi_{\xi}-\phi_{y}=q \psi_{\xi}-q \psi_{\xi}-\psi_{z},
$$

or

$$
\phi_{y}=\psi_{z},
$$

whose common value, regarding $\xi, y, z, p, q$ for the moment as the independent variables of our problem, must be an $f(\xi, p, q)$, for $\phi_{y}$ is free of $z$ and $\psi$. free of $y$. It follows that

$$
\begin{aligned}
& \phi=\omega_{p}=f(\xi, p, q) y+g(\xi, p, q), \\
& \psi=\omega_{q}=f(\xi, p, q) z+h(\xi, p, q) .
\end{aligned}
$$

We apply now the condition $\omega_{p q}=\omega_{q p}$, which gives

$$
f_{\xi} y z+f_{q} y+g_{\xi} z+g_{q}=f_{\xi} y z+f_{p} z+h_{\xi} y+h_{p} ;
$$

and therefore, since (52) must hold identically in $\xi, y, z, p, q$,

$$
f_{q}=h_{\xi}, \quad g_{\xi}=f_{p}, \quad g_{q}=h_{p} .
$$

This implies the existence of a $\lambda(\xi, p, q)$ such that

$$
f=\lambda_{\xi}, \quad g=\lambda_{p}, \quad h=\lambda_{q} .
$$

Thus (50), (51) become

$$
\begin{aligned}
& \omega_{p}=\lambda_{\xi} y+\lambda_{p}, \\
& \omega_{q}=\lambda_{\xi} z+\lambda_{q} .
\end{aligned}
$$

Going back to $x, y, z, p, q$ as independent variables in $\lambda$, the second members of (55), (56) are respectively $\partial \lambda / \partial p, \partial \lambda / \partial q$; hence the general solution of these equations is

$$
\omega=\lambda(\xi, p, q)-\mu(x, y, z),
$$

where $\mu$ is an arbitrary function of its arguments. 
By this and (44) we have

$$
\tau-\lambda(\xi, \phi ; q)=\sigma-\mu(x, y, z) .
$$

Since, as is readily seen, the modification of $\tau$ by the subtractive $\lambda(\xi, p, q)$ does not affect the values of the second members of (39), nor the modification of $\sigma$ by the subtractive $\mu(x ; y, z)$ the values of the second members of (38), if we denote by $V(x, y, z, p, q)$ the common value of the two members of (58), we have

$$
\begin{array}{ll}
\frac{Y}{M}=V_{p}, & \frac{Z}{M}=V_{q}, \\
\frac{P}{M}=p V_{x}-V_{y}, & \frac{Q}{M}=q V_{z}-V_{z},
\end{array}
$$

to which we may adjoin

$$
\frac{X}{M}=1-p V_{p}-q V_{q}
$$

by the definition (33) of $M$.

The existence of this $V$ established, we can construct

$$
W=e^{v}, \quad \rho=M e^{-v} \text {. }
$$

Substituting

$$
V=\log W, \quad M=\rho W,
$$

in (59), $\left(59^{\prime}\right)$, we obtain the system (32). Thus the proof is completed that the conditions (34) are necessary and sufficient for the existence of a $W$ and a $\rho$ verifying (32).

\section{ANALYTIC SOLUTION OF THE PROBLEM}

Our problem is now the following: To prove that if a given infinitesimal element transformation $\epsilon$

$$
\begin{array}{lll}
\frac{\delta x}{\delta t}=X(x, y, z, p, q), & \frac{\delta y}{\delta t}=Y(x, y, z, p, q), & \frac{\partial z}{\delta t}=Z(x, y, z, p, q), \\
\frac{\delta p}{\delta t}=P(x, y, z, p, q), & \frac{\delta q}{\delta t}=Q(x, y, z, p, q),
\end{array}
$$

applied repeatedly to the elements of an arbitrary surface $\Sigma$ generates a field of $\infty^{3}$ elements which is integrable, then $\epsilon$ must obey the system (34).

Denoting by the subscript 0 quantities relative to $\Sigma$, we write the equation of that surface in the form 


$$
x_{0}=-\phi\left(y_{0}, z_{0}\right) .
$$

Its $\infty^{2}$ surface elements are expressed in terms of the two parameters $y_{0}, z_{0}$ by the equations

(64) $x_{0}=-\phi\left(y_{0}, z_{0}\right), \quad y_{0}=y_{0}, \quad z_{0}=z_{0}, \quad p_{0}=\phi_{y 0}\left(y_{0}, z_{0}\right), \quad q_{0}=\phi_{z_{0}}\left(y_{0}, z_{0}\right)$.

We may regard (62) as a system of differential equations for $x, y, z, p, q$ as functions of $t$. Let the uniquely determined solution of this system which takes the values (64) for $t=0$ be denoted by

$$
\begin{array}{lll}
x=x\left(t, y_{0}, z_{0}\right), & y=y\left(t, y_{0}, z_{0}\right), \quad z=z\left(t, y_{0}, z_{0}\right), \\
p=p\left(t, y_{0}, z_{0}\right), & q=q\left(t, y_{0}, z_{0}\right) .
\end{array}
$$

These equations represent in terms of the three parameters $t, y_{0}, z_{0}$ the field of $\infty^{3}$ elements generated from $\Sigma$ by means of $\epsilon$. The condition that this field be integrable is

$$
(p x y)-(q z x)+p(q y z)-q(p y z)=0, *
$$

where the parentheses denote jacobians as to $t, y_{0}, z_{0}$.

Using (62), this can be expanded into

$$
\begin{aligned}
& P(x y)+X(y p)+Y(p x)-Q(z x)-Z(x q)-X(q z) \\
& \quad+p Q(y z)+p Y(z q)+p Z(q y)-q P(y z)-q Y(z p)-q Z(p y)=0,
\end{aligned}
$$

where the parentheses denote determinants in the matrix

$$
\left|\begin{array}{lllll}
\frac{\partial x}{\partial y_{0}} & \frac{\partial y}{\partial y_{0}} & \frac{\partial z}{\partial y_{0}} & \frac{\partial p}{\partial y_{0}} & \frac{\partial q}{\partial y_{0}} \\
\frac{\partial x}{\partial z_{0}} & \frac{\partial y}{\partial z_{0}} & \frac{\partial z}{\partial z_{0}} & \frac{\partial p}{\partial z_{0}} & \frac{\partial q}{\partial z_{0}}
\end{array}\right| .
$$

We introduce to represent these determinants ten symbols $\omega$ as follows:

$$
\begin{aligned}
& \omega_{1}=(x y), \quad \omega_{2}=(x z), \quad \omega_{3}=(x p), \quad \omega_{4}=(x q), \quad \omega_{5}=(y z), \\
& \begin{array}{lll}
\omega_{6}=(y p), & \omega_{7}=(y q), \quad \omega_{8}=(z p), \quad \omega_{9}=(z q), \quad \omega_{10}=(p q) .
\end{array}
\end{aligned}
$$

Formula (67) can then be abbreviated to

$$
\sum A_{i} \omega_{i}=0
$$

by means of the further notation

(71) $\begin{array}{ll}A_{1} & =P, \quad A_{2}=Q, \quad A_{3}=-Y, A_{4}=-Z, \quad A_{5}=p Q-q P, \\ A_{6} & =X+q Z, A_{7}=-p Z, A_{8}=-q Y, A_{9}=X+p Y, A_{10}=0 .\end{array}$

* This condition is obtained by making the change of variables (65) in the Pfaff equation $d x+p d y+q d z=0$, and then applying the usual criterion for exactness to the resulting Pfaffian in $\ell, y_{0}, z_{0}$. 
In (70) and everywhere hereafter the index runs from 1 to 10.

Denoting by means of a bar the partial derivatives of $x, y, z, p, q$ as to $y_{0}$, we see that these obey the equations of variation of (62):

$$
\begin{aligned}
& \bar{x}^{\prime}=X_{x} \bar{x}+X_{y} \bar{y}+X_{z} \bar{z}+X_{p} \bar{p}+X_{q} \bar{q}, \\
& \cdot \cdot \cdot \cdot \cdot \cdot \cdot \cdot \cdot \\
& \bar{q}^{\prime}=Q_{x} \bar{x}+Q_{y} \bar{y}+Q_{z} \bar{z}+Q_{p} \bar{p}+Q_{q} \bar{q},
\end{aligned}
$$

obtained by substituting (65) in (62) and differentiating the resulting identities in $t, y_{0}, z_{0}$ partially as to $y_{0}$. The accent denotes differentiation as to $t$ with $y_{0}, z_{0}$ held fixed, that is, differentiation along a path curve of $\epsilon$.

Evidently the partial derivatives of $x, y, z, p, q$ as to $z_{0}$ obey the same equations of variation.

From this it follows that the determinants $\omega$ obey a system of linear differential equations. Consider for instance $\omega_{1}=(x y)$. We have

$$
\begin{aligned}
\omega_{1}^{\prime}= & \left(x^{\prime} y\right)+\left(x y^{\prime}\right) \\
= & X_{x}(x y)+X_{z}(z y)+X_{p}(p y)+X_{q}(q y) \\
& \quad+Y_{y}(x y)+Y_{z}(x z)+Y_{p}(x p)+Y_{q}(x q)
\end{aligned}
$$

by (72), or

(74) $\omega_{1}^{\prime}=\left(X_{x}+Y_{y}\right) \omega_{1}+Y_{z} \omega_{2}+Y_{p} \omega_{3}+Y_{Q} \omega_{4}-X_{8} \omega_{5}-X_{p} \omega_{6}-X_{Q} \omega_{7}$.

Similarly each $\omega^{\prime}$ can be expressed as a homogeneous linear form in the $\omega$ 's. Carrying the calculations through, we obtain the system of linear differential equations represented by the following scheme (75):

\begin{tabular}{c|c|c|c|c|c|c|c|c|c|c}
\hline & $\omega_{1}$ & $\omega_{z}$ & $\omega_{3}$ & $\omega_{4}$ & $\omega_{5}$ & $\omega_{8}$ & $\omega_{7}$ & $\omega_{8}$ & $\omega_{9}$ & $\omega_{10}$ \\
\hline$\omega_{1}^{\prime}$ & $X_{x}+Y_{y}$ & $Y_{z}$ & $Y_{p}$ & $Y_{q}$ & $-X_{z}$ & $-X_{p}$ & $-X_{q}$ & & & \\
\hline$\omega_{z}^{\prime}$ & $Z_{y}$ & $X_{x}+Z_{z}$ & $Z_{p}$ & $Z_{q}$ & $X_{y}$ & & & $-X_{p}$ & $-X_{q}$ & \\
\hline$\omega_{z}^{\prime}$ & $P_{y}$ & $P_{z}$ & $X_{x}+P_{p}$ & $P_{q}$ & & $X_{y}$ & & $X_{z}$ & & $-X_{q}$ \\
\hline$\omega_{q}^{\prime}$ & $Q_{y}$ & $Q_{z}$ & $Q_{p}$ & $X_{x}+Q_{q}$ & & & $X_{y}$ & & $X_{z}$ & $X_{p}$ \\
\hline$\omega_{6}^{\prime}$ & $-Z_{x}$ & $Y_{x}$ & & & $Y_{y}+Z_{z}$ & $Z_{p}$ & $Z_{q}$ & $-Y_{p}$ & $-Y_{q}$ & \\
\hline$\omega_{6}^{\prime}$ & $-P_{x}$ & & $Y_{x}$ & & $P_{z}$ & $Y_{y}+P_{p}$ & $P_{q}$ & $Y_{z}$ & & $-Y_{q}$ \\
\hline$\omega_{7}^{\prime}$ & $-Q_{x}$ & & & $Y_{x}$ & $Q_{z}$ & $Q_{p}$ & $Y_{y}+Q_{q}$ & & $Y_{z}$ & $Y_{p}$ \\
\hline$\omega_{8}^{\prime}$ & & $-P_{x}$ & $Z_{x}$ & & $-P_{y}$ & $Z_{y}$ & & $Z_{z}+P_{p}$ & $P_{q}$ & $-Z_{q}$ \\
\hline$\omega_{q}^{\prime}$ & & $-Q_{x}$ & & $Z_{x}$ & $-Q_{y}$ & & $Z_{y}$ & $Q_{p}$ & $Z_{z}+Q_{q}$ & $Z_{p}$ \\
\hline$\omega_{10}^{\prime}$ & & & $-Q_{x}$ & $P_{x}$ & & $-Q_{y}$ & $P_{y}$ & $-Q_{z}$ & $P_{z}$ & $P_{p}+Q_{q}$ \\
\hline \hline
\end{tabular}


As a solution of the system (75), the $\omega$ 's will be completely determined if we know their values for $t=0$, that is, on the base surface $\Sigma$. The values on $\Sigma$ of the elements of the matrix (68) are evidently the following:

$$
\left|\begin{array}{lllll}
-p_{0} & 1 & 0 & r_{0} & s_{0} \\
-q_{0} & 0 & 1 & s_{0} & t_{0}
\end{array}\right|
$$

where $r_{0}, s_{0}, t_{0}$ denote the second partial derivatives of the function $\phi$ which defines $\Sigma$.

Hence the values of the $\omega$ 's on $\Sigma$, which values we denote by the symbols $\alpha$, are

$$
\begin{aligned}
& \alpha_{1}=q_{0}, \quad \alpha_{2}=-p_{0}, \quad \alpha_{8}=-p_{0} s_{0}+q_{0} r_{0}, \quad \alpha_{4}=-p_{0} t_{0}+q_{0} s_{0}, \\
& \alpha_{6}=1, \quad \alpha_{6}=s_{0}, \quad \alpha_{7}=t_{0}, \quad \alpha_{8}=-r_{0}, \\
& \alpha_{9}=-s_{0}, \quad \alpha_{10}=r_{0} t_{0}-s_{0}^{2} .
\end{aligned}
$$

The necessary and sufficient condition that the field of elements generated from $\Sigma$ by means of $\epsilon$ be integrable is that the ten w's which satisfy (75) and take for $t=0$ the values (77) shall obey the linear relation (70), and that identically in the parameters $y_{0}, z_{0}$.

The analytic expression of this condition is a problem which we have already solved in a previous paper.*

Let us denote by $\Omega$ the left member of (70):

$$
\Omega=\sum_{i} A_{i} \omega_{i} .
$$

The $\omega$ 's are functions of $t, y_{0}, z_{0}$, and the $A$ 's functions of $x, y, z, p$, which after the substitution (65) also become functions of $t, y_{0}, z_{0}$. Assuming, as we do, that all functions involved in our considerations are analytic, the necessary and sufficient condition for the identical vanishing of $\Omega$ is that $\Omega$ together with its derivatives of all orders vanish for $t=0$, identically in $y_{0}, z_{0}$.

Using after each successive differentiation of $\Omega$ the system (75), which we abbreviate to

$$
\omega_{i}^{\prime}=\sum_{i} p_{i j} \omega_{i},
$$

we find that each derivative $\Omega^{\prime}, \Omega^{\prime \prime}, \ldots$ is, like $\Omega$ itself, a homogeneous linear form in the $\omega$ 's. We obtain, in fact,

$$
\Omega=\sum_{i} A_{i} \omega_{i}, \quad \Omega^{\prime}=\sum_{i} B_{i} \omega_{i}, \quad \Omega^{\prime \prime}=\sum_{i} C_{i} \omega_{i}, \cdots,
$$

* Normal congruences . . . , previously cited; see $\$ 5$ of that paper. 
where each set of ten symbols $B, C, \cdots$ is derived from its predecessor the recurrence formulas

$$
\begin{aligned}
& B_{i}=A_{i}^{\prime}+\sum_{j} p_{j i} A_{i}, \\
& C_{i}=B_{i}^{\prime}+\sum_{j} p_{i i} B_{i},
\end{aligned}
$$

in which the accent denotes $d / d t$ along a path curve of $\epsilon$, that is

$$
, \equiv X \frac{\partial}{\partial x}+Y \frac{\partial}{\partial y}+Z \frac{\partial}{\partial z}+P \frac{\partial}{\partial p}+Q \frac{\partial}{\partial q} .
$$

The quantities $B, C, \cdots$ are thus expressions of higher and higher order in the partial derivatives of $X, Y, Z, P, Q$.

In demanding that $\Omega, \Omega^{\prime}, \Omega^{\prime \prime}, \ldots$ all vanish for $t=0$, we get the infinite sequence of conditions

$$
\sum_{i} A_{i} \alpha_{i}=0, \quad \sum_{i} B_{i} \alpha_{i}=0, \quad \sum_{i} C_{i} \alpha_{i}=0, \cdots,
$$

where the $A, B, C, \cdots$ are the values for $t=0$ of the $A, B, C, \cdots$ of (79); in other words, the $A, B, C, \cdots$ of (81) are the values on $\Sigma$ of the same letters of (79), and are therefore to be derived from the latter by attaching the subscript 0 to $x, y, z, p, q$.

Substituting the values (77) of the $\alpha$ 's in (81), we obtain, after dropping the subscript 0 , the following infinite system of partial differential equations of the Monge-Ampère type:

$$
\begin{aligned}
& \mathfrak{B}_{0}\left(r t-s^{2}\right)+\mathfrak{B}_{1} r+\mathfrak{B}_{2} s+\mathfrak{B}_{3} t+\mathfrak{B}_{4}=0, \\
& \mathfrak{E}_{0}\left(r t-s^{2}\right)+\mathfrak{C}_{1} r+\mathfrak{C}_{2} s+\mathfrak{C}_{3} t+\mathfrak{C}_{4}=0,
\end{aligned}
$$

where the symbols $\mathfrak{B}, \mathfrak{C}, \cdots$ are derived respectively from $B, C, \cdots$ by the same formulas, typified by

$$
\begin{aligned}
& \mathfrak{B}_{0}=B_{10}, \\
& \mathfrak{B}_{1}=q B_{3}-B_{8}, \\
& \mathfrak{B}_{2}=-p B_{3}+q B_{4}+B_{6}-B_{8}, \\
& \mathfrak{B}_{3}=-p B_{4}+B_{7}, \\
& \mathfrak{B}_{4}=q B_{1}-p B_{2}+B_{5} .
\end{aligned}
$$


The equation $\left(82_{0}\right), \mathfrak{A}_{0}\left(r t-s^{2}\right)+\cdots$, disappears identically, that is, each $\mathfrak{A}=0$, as a consequence of the fact that $\Sigma$ itself is always an integral surface of the field of elements which it generates.

$A$ surface $\Sigma$ gives rise to an integrable element-field when and only when it obeys the infinite system (82).

If an arbitrary surface $\Sigma$ gives an integrable element-field, each equation (82) must disappear identically. In particular $\left(82_{1}\right)$ must do so, that is,

$$
\mathfrak{B}_{0}=0, \quad \mathfrak{B}_{1}=0, \quad \mathfrak{B}_{2}=0, \quad \mathfrak{B}_{3}=0, \quad \mathfrak{B}_{4}=0 .
$$

Let us calculate the explicit values of the $B$ 's by carrying out the operations indicated in (80). We find, with reference to (71) and the scheme (75),

$$
\begin{aligned}
& B_{1}=\left\{P X_{x}+(q P-p Q) Z_{x}-q Z P_{x}+p Z Q_{x}\right\}+\left(P Y_{y}+Q Z_{y}-Z Q_{y}\right) \\
& +Z P_{z}+P P_{p}+Q P_{q} \text {, } \\
& B_{2}=\left\{Q X_{x}+(p Q-q P) Y_{x}+q Y P_{x}-p Y Q_{x}\right\}+Y Q_{y} \\
& +\left(P Y_{z}+Q Z_{z}-Y P_{z}\right)+P Q_{p}+Q Q_{q} \text {, } \\
& B_{3}=\left(-Y X_{x}+q Z Y_{x}-q Y Z_{x}\right)-Y Y_{y}-Z Y_{s} \\
& +\left(Q Z_{p}-Y P_{p}-Z Q_{p}\right)-Q Y_{q} \\
& B_{4}=\left(-Z X_{x}-p Z Y_{x}+p Y Z_{x}\right)-Y Z_{y}-Z Z_{x} \\
& -P Z_{p}+\left(P Y_{q}-Y P_{q}-Z Q_{q}\right) \text {, } \\
& B_{5}=\left(-q X P_{x}+p X Q_{x}\right)+\left\{Q X_{y}+(p Q-q P) Y_{y}-X Q_{y}\right\} \\
& +\left\{-P X_{z}+(p Q-q P) Z_{z}+X P_{z}\right\} \\
& +\left(-q P P_{p}+p P Q_{p}\right)+\left(-q Q P_{q}+p Q Q_{q}\right) \text {, }
\end{aligned}
$$

(85) $B_{6}=Z Q+\left(X X_{x}+q X Z_{x}\right)+(X+q Z) Y_{y}+\left(Z X_{z}+q Z Z_{z}\right)$

$$
+\left\{p Q Z_{p}+(X+q Z) P_{p}-p Z Q_{p}\right\}+\left(Q X_{q}+q Q Z_{q}\right),
$$

$$
\begin{aligned}
& B_{7}=-Z P-p X Z_{x}+\left(-Z X_{y}-p Z Y_{y}+X Z_{y}\right)-p Z Z_{z} \\
&-p P Z_{p}+\left\{-P X_{q}-q P Z_{q}+(X+q Z) P_{q}-p Z Q_{q}\right\}, \\
& B_{8}=-Y Q-q X Y_{x}-q Y Y_{y}+\left(-Y X_{z}+X Y_{z}-q Y Z_{z}\right) \\
& \quad+\left\{-Q X_{p}-p Q Y_{p}-q Y P_{p}+(X+p Y) Q_{p}\right\}-q Q Y_{q},
\end{aligned}
$$




$$
\begin{gathered}
B_{9}=Y P+\left(X X_{x}+p X Y_{x}\right)+\left(Y X_{y}+p Y Y_{y}\right)+(X+p Y) Z_{z} \\
+\left(P X_{p}+p P Y_{p}\right)+\left\{g P Y_{q}-q Y P_{q}+(X+p Y) Q_{q}\right\} \\
B_{10}=\left\{-Z X_{p}-p Z Y_{p}+(X+p Y) Z_{p}\right\}+\left\{Y X_{q}-(X+q Z) Y_{q}+q Y Z_{q}\right\}
\end{gathered}
$$

From these we can then determine by means of (83) the values of the symbols $\mathfrak{B}$; they can be written, using $M=X+p Y+q Z$,

$$
\begin{aligned}
& \mathfrak{B}_{0}=\left(M Z_{p}-Z M_{p}\right)-\left(M Y_{q}-Y M_{q}\right) \\
& \mathfrak{B}_{1}=q\left(M Y_{x}-Y M_{x}\right)-\left(M Y_{z}-Y M_{z}\right)-\left(M Q_{p}-Q M_{p}\right) \\
& \mathfrak{B}_{2}=-p\left(M Y_{x}-Y M_{x}\right)+q\left(M Z_{x}-Z M_{x}\right)+\left(M Y_{y}-Y M_{y}\right) \\
& \quad-\left(M Z_{z}-Z M_{z}\right)+\left(M P_{p}-P M_{p}\right)-\left(M Q_{q}-Q M_{q}\right) \\
& \mathfrak{B}_{3}=-p\left(M Z_{x}-Z M_{x}\right)+\left(M Z_{y}-Z M_{y}\right)+\left(M P_{q}-P M_{q}\right) \\
& \mathfrak{B}_{4}=-q\left(M P_{x}-P M_{x}\right)+p\left(M Q_{x}-Q M_{x}\right) \\
& \quad-\left(M Q_{y}-Q M_{y}\right)+\left(M P_{z}-P M_{z}\right) .
\end{aligned}
$$

After division by $M^{2}$, the five conditions $\mathfrak{B}=0$ are seen to be identical with the system (34), previously proved necessary and sufficient in order that $\epsilon$ be proportional to an infinitesimal contact transformation. Hence our main theorem.

\section{STRONGER FORMS OF STATEMENT}

The theorem was derived from the conditions $\mathscr{B}=0$ alone, instead of from the complete infinite system $\mathfrak{B}=0, \mathfrak{E}=0, \cdots$. The identical disappearance of only the first equation (82) signifies that the element-field generated from $\Sigma$ by $\epsilon$ has, besides $\Sigma$, an integral surface consecutive to $\Sigma$. We may therefore strengthen our theorem by substituting this requirement in its hypothesis instead of that of the existence of $\infty^{1}$ integral surfaces.

The other stronger form stated in the introduction, in which certain $5 \infty^{3}$ manifolds are substituted for the $\infty^{\infty}$ manifolds $\Sigma$, results from the following observations.

We may regard points and planes as spheres of radius zero and infinity respectively. For a sphere of radius $R$ we have

$$
\frac{r}{1+p^{2}}=\frac{s}{p q}=\frac{t}{1+q^{2}}=\frac{\left(1+p^{2}+q^{2}\right)^{1 / 2}}{R}
$$


Substituting the resulting values of $r, s, t$ in $\left(82_{1}\right)$, we obtain a quadratic equation in $R$. If this equation is satisfied by $R=0$ and $R=\infty$, the constant term and the coefficient of $R^{2}$ must reduce to zero, that is

$$
\mathfrak{B}_{0}=0, \quad \mathfrak{B}_{4}=0 .
$$

Again, for a curve regarded as the limit as $R \rightarrow 0$ of a canal surface generated by a sphere of radius $R$, we can calculate that $r, s, t$ become infinite in the ratios

$$
r: s: t=z^{\prime 2}:-y^{\prime} z^{\prime}: y^{\prime 2}
$$

where $1: y^{\prime}: z^{\prime}$ defines the direction of the curve at the particular point under consideration.

Under $(88)$ the equation $\left(82_{1}\right)$ reduces to

$$
\mathfrak{B}_{1} r+\mathfrak{B}_{2} s+\mathfrak{B}_{3} t=0 .
$$

This is satisfied by a curve $C$ when and only when

$$
\mathfrak{B}_{1} z^{\prime 2}-\mathscr{B}_{2} y^{\prime} z^{\prime}+\mathfrak{B}_{3} y^{\prime 2}=0
$$

where the $(x, y, z, p, q)$ in $\mathfrak{B}_{1}, \mathfrak{B}_{2}, \mathfrak{B}_{3}$ are the coördinates of an arbitrary surface element of $C$ and $1: y^{\prime}: z^{\prime}$ defines the direction element of $C$ which is contained by that surface element.

Now, if in every surface element $(x, y, z, p, q)$ of space there are three distinct directions which belong to curves that give rise to integrable fields, then (91) must have for given $(x, y, z, p, q)$ three solutions for the ratio $y^{\prime}: z^{\prime}$. Being a quadratic equation, (91) must therefore disappear identically, that is

$$
\mathfrak{B}_{1}=0, \quad \mathfrak{B}_{2}=0, \quad \mathfrak{B}_{3}=0 .
$$

Adjoined to (88), these complete the five conditions (84) which are necessary and sufficient in order that $\epsilon$ be proportional to a contact transformation.

Since in the last discussion we have made use of only the first equation of (82), it is sufficient to require that each of the $5 \infty^{3}$ manifolds specified give rise to an element-field having an integral surface consecutive to its base manifold.

Pronceton University, Prnnceton, N. J. 